\title{
Comparison of proliferation and differentiation potential between mouse primary hepatocytes and embryonic hepatic progenitor cells in vitro
}

\author{
YUN HE ${ }^{*}$, JIAN-WU ZHOU* , LEI XU, MENG-JIA GONG, TONG-CHUAN HE and YANG BI \\ Department of Pediatric Surgery, Stem Cell Biology and Therapy Laboratory, Ministry of Education \\ Key Laboratory of Child Development and Disorders, Chongqing Stem Cell Therapy Engineering Technical Center, \\ The Children's Hospital of Chongqing Medical University, Chongqing 400014, P.R. China
}

Received March 7, 2013; Accepted May 22, 2013

DOI: 10.3892/ijmm.2013.1413

\begin{abstract}
Cell therapy may be a novel and effective treatment strategy for liver diseases, replacing liver transplantation. The potential of two alternative cell types (hepatic progenitor/stem cells and mature hepatocytes) has not yet been fully assessed; the issues of low amplification efficiency and recovery function remain to be resolved. In this study, we investigated the proliferation, differentiation and function of primary mouse mature hepatocytes and embryonic hepatic progenitor cells. Primary cells were obtained from the livers of mouse embryos at 14.5 days post coitus [hepatic progenitor $14.5 \mathrm{~d}$ (HP14.5d) cells], as well as from the livers of 3-month-old mice [liver cells $3 \mathrm{~m}$ (LC3m)]. Using trypan blue staining and crystal violet staining to detect cell viability, we found that compared with the limited growth capability of primary LC3m cells, primary HP14.5d cells exhibited an active cell proliferation; however, proliferative ability of passaged HP14.5d cells significantly decreased. After the HP14.5d cells were treated in hepatic induction medium, the expression of progenitor cell markers decreased and that of mature hepatic markers increased, to levels similar to those of LC3m cells. On day 12 of induction, the HP14.5d cells showed comparable indocyanine green (ICG) uptake and glycogen storage to that of the LC $3 \mathrm{~m}$ cells. Therefore, our study demonstrates that primary hepatic progenitor cells have a stronger proliferation capacity and differentiation potential, supporting their clinical application in liver cell transplantation.
\end{abstract}

Correspondence to: Dr Yang Bi, Stem Cell Biology and Therapy Laboratory, The Children's Hospital of Chongqing Medical University, Building 7, Room 905, 136 Zhongshan Er Road, Chongqing 400014, P.R. China

E-mail: yangbi1981@yahoo.com

*Contributed equally

Key words: hepatocyte, hepatic progenitor cells, proliferation, differentiation

\section{Introduction}

Orthotopic liver transplantation (OLT) is one of the best and most effective methods for the treatment of end-stage liver disease or liver-based metabolic diseases (1-3). However, due to the dramatic imbalance between the limited number of available donors and the patients who require transplantation, an increasingly high number of patients are unable to find a suitable liver for transplantation. On the other hand, the complications associated with OLT surgery hamper its clinical application $(4,5)$. Several studies have demonstrated that cell-based transplantation strategies have great potential for repair and functional recovery following acute liver damage, becoming a realistic option for the treatment of liver diseases (6-8).

A reliable cell source is required for this effective clinical therapy. Isolated mature hepatocytes are difficult to manipulate and cannot be expanded in vitro to obtain a large cell population. The viability of these cells and hepatic markers are easily lost under common culture conditions in vitro (9). Certain studies have found that hepatic stem cells (HSCs) have a multilineage differentiation potential, as well as selfproliferative capabilities and can differentiate into a mature hepatic cell lineage; furthermore, they have been shown to exhibit certain phenotypes and functions characteristic of hepatocytes in vitro (10). Moreover, HSCs showing a greater regenerative capacity than adult hepatocytes, participate in liver tissue repair and reconstruction following injury. It has been reported that HSC transplantation may be utilized to substitute OLT, having a definite therapeutic effect on patients with end-stage liver disease $(11,12)$.

Thus far, there are no definite distinguishing features as regards HSCs. Most scholars hypothesize that HSCs should be composed of two main categories, extrahepatic and intrahepatic stem cells. The former includes embryonic stem cells, hematopoietic stem cells and bone marrow mesenchymal stem cells. The latter includes embryonic HSCs, hepatic oval cells and small hepatocyte-like progenitor cells $(13,14)$. HSCs derived from the fetal liver, also known as hepatic progenitor cells (HPCs), exhibit bipotential capacity and are characterized by an intermediary phenotype between biliary epithelial cells and hepatocytes. HPCs act as an important liver cell 
source of hepatocyte transplantation and biological artificial liver $(15,16)$.

The present study aimed to investigate the phenotype, proliferation and differentiation capacity of primary embryonic HPCs compared with mature hepatocytes. HPCs exhibit better growth capability and function as mature hepatocytes following induction in vitro. The study of HPCs may aid in the understanding of the process of artificial liver development and promote its clinical application.

\section{Materials and methods}

Cells and chemicals. The Hepa1-6 line was purchased from the American Type Culture Collection (Manassas, VA, USA) and maintained in complete Dulbecco's modified Eagle's medium (DMEM) supplemented with $10 \%$ fetal bovine serum (FBS, HyClone, Logan, UT, USA), $100 \mathrm{U} / \mathrm{ml}$ penicillin, and $100 \mu \mathrm{g} / \mathrm{ml}$ streptomycin at $37^{\circ} \mathrm{C}$ in $5 \% \mathrm{CO}_{2}$. Unless indicated otherwise, all chemicals were purchased from Sigma-Aldrich (St. Louis, MO, USA). Hepatic differentiation induction medium was composed of DMEM supplemented with $2 \%$ horse serum (HS), $0.1 \mu \mathrm{mol} / 1$ dexamethason (Dex), $10 \mathrm{ng} / \mathrm{ml}$ hepatic growth factor (HGF) and $20 \mathrm{ng} / \mathrm{ml}$ fibroblast growth factor 4 (FGF4).

Isolation of primary cells. Primary cells were obtained from the livers of mouse embryos at 14.5 days post coitus [hepatic progenitor 14.5d (HP14.5d) cells], as well as from the livers of 3-month-old mice [liver cells 3m (LC3m)]. Briefly, adult livers were perfused with phosphate-buffered saline (PBS) and collagenase type IV through the inferior vena cava, and then cut open to gently scrape off the cells. The embryronic livers were cut into small sections and digested with collagenase type IV at $37^{\circ} \mathrm{C}$ for $10 \mathrm{~min}$. Isolated cell clumps were dissociated by pipetting in $10 \mathrm{ml}$ DMEM, then the solution was filtered through a $100-\mu \mathrm{m}$ cell strainer and centrifuged at $50 \mathrm{x}$ g for $3 \mathrm{~min}$. The cells were gently resuspended in complete DMEM and plated on $100-\mathrm{mm}$ dishes that were coated with type I collagen and incubated at $37^{\circ} \mathrm{C}$. After $24 \mathrm{~h}$, all non-adherent cells were removed and the medium was changed every 3 days. The HP14.5d cells at $90 \%$ of confluence were trypsinized and passaged.

Cell viability. Trypan blue staining and crystal violet staining were performed to assess cell viability. As previously described (17), the cells were incubated in 24-well plates at $2.0 \times 10^{4}$ cells per well and cell viability was measured at the indicated time points. For trypan blue staining, both adherent and suspended cells were collected and mixed with $0.4 \% 2 \mathrm{X}$ trypan blue buffer (Beyotime, Nantong, China). A total of $10 \mu \mathrm{l}$ of cell mixture $\left(10^{6} \mathrm{cell} / \mathrm{s} / \mathrm{ml}\right)$ was added into the hemocytometer and observed under a microscope (TE2000-S; Nikon, Tokyo, Japan). Blue-stained cells were counted as dead cells. Three independent experiments were performed in duplicate. The mean and standard deviation were calculated. The viable cells were unstained, while dead cells were stained blue. For crystal violet staining, the culture medium was removed, the cells were fixed with $4 \%$ paraformaldehyde at room temperature for $10 \mathrm{~min}$ and then stained with $0.05 \%$ crystal violet for $30 \mathrm{~min}$. The cells were then washed with tap water, after which the water was removed and the cells were dried out on filter paper. After the plates were photographed, blue dye was dissolved in $500 \mu \mathrm{l}$ of methanol and emission spectra were measured at an excitation wavelength of $540 \mathrm{~nm}$ using a Multimode Microplate Reader (Thermo Fisher Scientific, Waltham, MA, USA). Three independent experiments were performed in duplicate. The mean and standard deviation were calculated.

Transfection of albumin (ALB) promoter-driven reporter gene and Gaussia luciferase reporter assay. The plasmid of the ALB promoter-driven luciferase reporter gene (pSEB-ALBGluc) was constructed in our previous study (18). The cells were engrafted on 24-well plates at an initial confluence of $60 \%$. The pSEB-ALB-Gluc plasmid was transfected into the cells using Lipofectamine ${ }^{\circledR} 2000$ (Invitrogen, Carslbad, CA, USA). After $48 \mathrm{~h}$, the cells were treated with hepatic differentiation induction medium for 12 days. Gaussia luciferase reporter assay was carried out at each indicated time point. Briefly, $20 \mu \mathrm{l}$ of cell medium were collected and mixed with $10 \mu \mathrm{l}$ of fresh prepared luciferin substrate solution. Gaussia luciferase acitivity was immediately measured using a Single Tube Multimode Reader (Promega, Madison, WI, USA). Each assay condition was performed in triplicate. Data are expressed as the means $\pm \mathrm{SD}$.

RNA isolation and semiquantitative reverse transcription (RT)-PCR analysis. As previously described $(18,19)$, total RNA was extracted by using an RNA Extraction kit (Bioteke Corp., Beijing, China) and reverse transcribed into cDNA using Superscript II reverse transcriptase (Invitrogen). The cDNA samples were 5- to 10-fold diluted and subjected to PCR amplification. The sequences of all primers listed in Table I were designed using the Primer3 program. A touchdown PCR protocol was performed under the following programmed conditions: $95^{\circ} \mathrm{C} \times 3 \mathrm{~min}$, and then $92^{\circ} \mathrm{C} \times 20 \mathrm{sec}$, $65^{\circ} \mathrm{C} \times 20 \mathrm{sec}, 72^{\circ} \mathrm{C} \times 20 \mathrm{sec}, 9$ cycles, with $1^{\circ} \mathrm{C}$ degree decrease per cycle, followed by $94^{\circ} \mathrm{C}$ x $20 \mathrm{sec}, 55^{\circ} \mathrm{C}$ x $20 \mathrm{sec}$, $72^{\circ} \mathrm{C} \times 20 \mathrm{sec}$ for $25-30$ cycles and $72^{\circ} \mathrm{C} \times 3 \mathrm{~min}$. The PCR products for RT-PCR were electrophoresed on a $1.5 \%$ agarose gel. Glyceraldehyde 3-phosphate dehydrogenase (GAPDH) expression in each sample was used to normalize the template concentration.

Western blot analysis. Cells were lysed in RIPA buffer with phenylmethylsulfonyl fluoride (PMSF) to extract the total protein. Approximately $20 \mu \mathrm{g}$ of total protein per lane was loaded onto a $10 \%$ SDS-polyacrylamide gel for electrophoretic separation. Thereafter, proteins were transferred onto a polyvinylidene fluoride (PVDF) membrane. The membrane was blocked with $5 \%$ fat-free skimmed milk at room temperature for $1 \mathrm{~h}$ and incubated with primary antibody to UDP glucuronosyltransferase 1 family, polypeptide A complex locus (UGT1A) and $\beta$-actin (Santa Cruz Biotechnology, Inc., Santa Cruz, CA, USA) at $4^{\circ} \mathrm{C}$ overnight. The membranes were then probed with the appropriate second antibody conjugated with horseradish peroxidase (Santa Cruz Biotechnology, Inc.) for $1 \mathrm{~h}$. The immunoreactive bands of protein were developed using enhanced chemiluminescent substrate (Kaiji Bio Co., Nanjing, China) and exposed using the G:BOX iChemi XR 
Table I. Primers used for RT-PCR.

Forward ( $\left.5^{\prime}-3^{\prime}\right)$

Reverse (5'-3')

\begin{tabular}{lll}
\hline GAPDH & GGCTGCCCAGAACATCAT & CGGACACATTGGGGGTAG \\
DLK & GCTGGGACGGGAAATTCT & AACCCAGGTGTGCAGGAG \\
CD34 & AGGGAAAGGCCAATGTGAC & CCACCCAACCAAATCACAG \\
AFP & ACGAGGAAAGCCCCTCAG & GCCATTCCCTCACCACAG \\
CK19 & GCCCTAGAGCAGGCCAAT & ATCTTGTCGCGCAAGTCC \\
ALB & CCAGACATTCCCCAATGC & CAAGTTCCGCCCTGTCAT \\
CK18 & CTGGGCTCTGTGCGAACT & ACAGAGCCACCCCAGACA \\
TAT & ACCTTCAATCCCATCCGA & TCCCGACTGGATAGGTAG \\
ApoB & CATGTGATCCCCACAGCA & TCCCAGGACCATGGAAAA \\
\hline
\end{tabular}

RT-PCR, reverse transcription PCR; GAPDH, glyceraldehyde 3-phosphate dehydrogenase; DLK, delta-like homolog; AFP, alpha fetoprotein; ALB, albumin; TAT, tyrosine aminotransferase; ApoB, apolipoprotein B.

gel documentation system (Syngene, Cambridge, UK). All the abovementioned steps were carried out at room temperature unless otherswise specified.

Immunofluorescence staining. The cells were fixed with methanol at $-20^{\circ} \mathrm{C}$ for $15 \mathrm{~min}$. They were then blocked with $5 \%$ goat serum for $1 \mathrm{~h}$. Subsequently, the cells were incubated with delta-like homolog (DLK), alpha fetoprotein (AFP), ALB, UGT1A and CK18 primary antibody (Santa Cruz Biotechnology, Inc.) at $4^{\circ} \mathrm{C}$ overnight, followed by probing with DyLight ${ }^{\circledast}$ 594- or 488-conjugated secondary antibody (Jackson ImmunoResearch Laboratories, Inc., West Grove, PA, USA) for $30 \mathrm{~min}$. The nuclei were stained with DAPI. The presence of proteins was ascertained under a fluorescence microscope (TE2000-S; Nikon). The cells were washed twice with PBS after each step; all the abovementioned steps were carried out at room temperature unless otherwise specified.

Indocyanine green (ICG) uptake and release. The cultured cells in 24-well plates were washed twice with PBS and incubated with DMEM supplemented with ICG at a final concentration of $1 \mathrm{mg} / \mathrm{ml}$ for $1 \mathrm{~h}$ at $37^{\circ} \mathrm{C}$ in $5 \% \mathrm{CO}_{2}$. After DMEM was removed and the cells were gently washed several times with PBS, green-stained cells were photographed under a microscope. Complete medium was then added and the cells were incubated for $>6 \mathrm{~h}$; the cells were then observed under a microscope to ensure ICG release. At least 10 non-overlapping fields of vision were recorded.

Periodic acid-Schiff (PAS) staining. The cells were cultured in 24-well plates as described above. Paraformaldehyde (4\%) was added to fix the cells for $10 \mathrm{~min}$, followed by incubation with $0.5 \%$ periodic acid solution for $5 \mathrm{~min}$. After rinsing with tap water, the cells were incubated in Schiff's solution for $15 \mathrm{~min}$ and counterstained with hematoxylin solution for $2 \mathrm{~min}$, and finally rinsed with flowing water for clarification. All steps were carried out at room termperature. The positive cells were stained purple. More than 10 non-overlapping fields of vision in each group were recorded under a microscope.
Statistical analysis. All data are presented as the means \pm standard deviation (SD) and calculated using SPSS 15.0 statistic software. A two-tailed Student's t-test was used to evaluate significant differences between 2 groups. A P-value $<0.05$ was considered to indicate a statistically significant difference.

\section{Results}

Morphology of primary and passaged cells. As shown in Fig. 1, we found that the primary LC $3 \mathrm{~m}$ cells were gathered together to form sac-like glands and exhibited a typical cubic cell shape of hepatocytes. The confluence of LC $3 \mathrm{~m}$ cells did not differ between days 1 and 3 after induction; few of the LC3m cells were adherent following passage. The primary HP14.5d cells displayed cluster growth and were typically mononucleate with a single, spherical, central nucleus and high nucleus/cytoplasm (N/C) ratio; however, a quarter of them were binucleate. Following passage, the majority of the HP14.5d cells exhibited an elongated morphology. These results indicate that the morphology of HPCs is not completely similar to that of mature hepatocytes; the passaged HP14.5d cells displayed different cell shapes; possibly due to the cell diversity of the primary isolated cells.

Proliferation of primary and passaged cells. Due to the difference in cell growth status between the HP14.5d and LC $3 \mathrm{~m}$ cells, we wished to further detect the cell proliferation within 9 days of incubation following isolation or passage. Compared with the non-proliferative LC $3 \mathrm{~m}$ cells, the primary HP14.5d cells proliferated actively. The number of HP14.5d cells on day 7 increased by $>5$-fold, but slighlty decreased on day 9 due to limited growth space in the plate. The number of viable HP14.5d cells at passage (P)1 was significantly lower than the number of viable primary HP14.5d cells. The cell proliferation of the HP14.5d cells at P2 was almost the same as that of the primary LC3m cells (Fig. 2A). The number of crystal violet-stained cells was similar to the number of viable cells (Fig. 2B and C). Therefore, the low success rate of primary culture of mature hepatocytes may due to their limited growth 

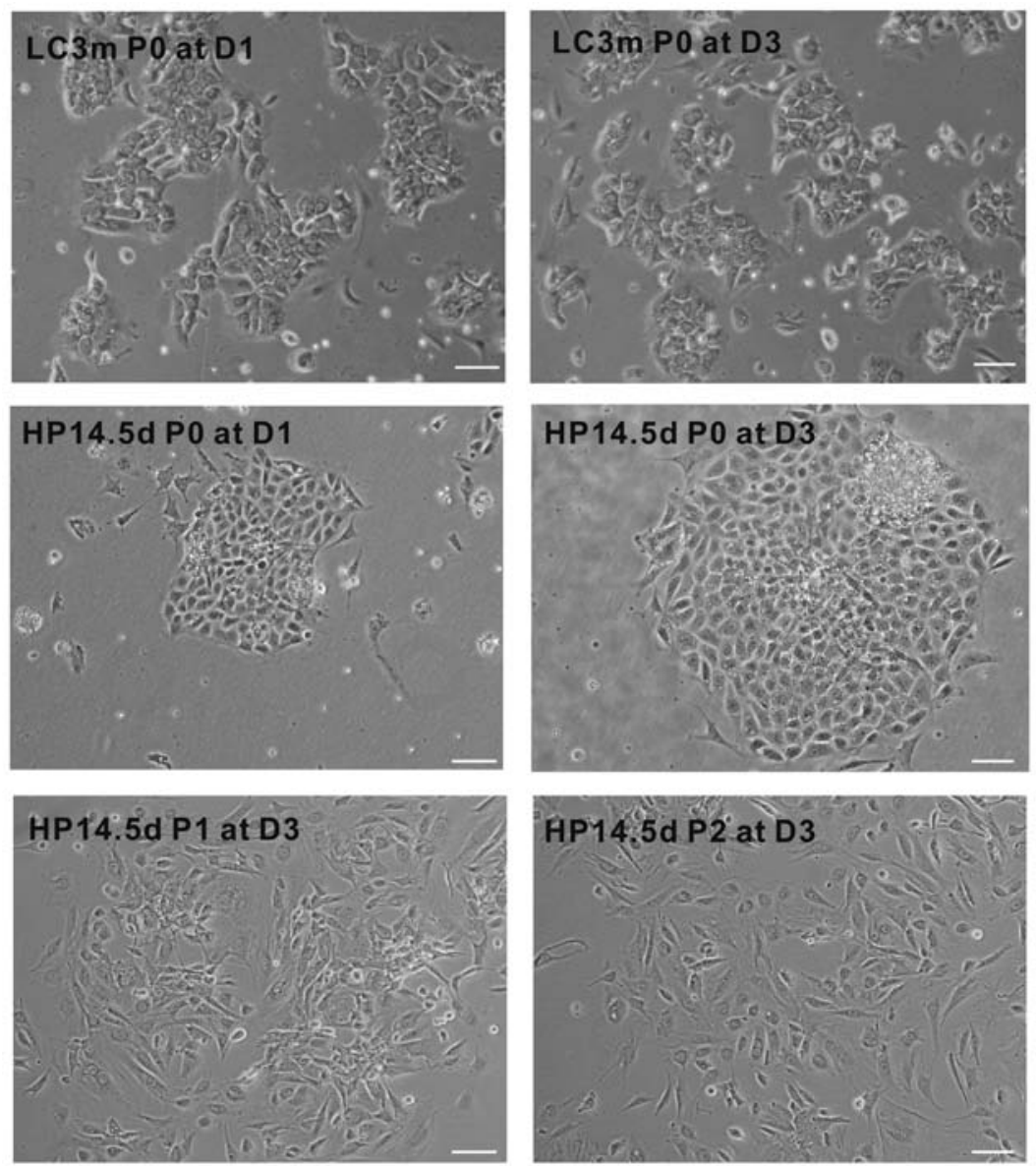

Figure 1. Morphology of primary and passaged cells. Primary cells were incubated with complete DMEM in 100-mm dishes, and the HP14.5d cells were trypsinized and passaged at a confluence of $90 \%$. P, passage; D, day. Scale bar, $200 \mu \mathrm{m}$

capabilities. HPCs primarily display active proliferation; however, their characteristics of proliferation and the hepatic phenotype become unstable following culture in vitro.

Identification of HP14.5d and LC3m cells. The expression of hepatic progenitor markers and mature hepatocyte markers was examined to identify the HP14.5d and LC3m cells. As shown by the RT-PCR and immunofluorescence staining results (Fig. 3), the early hepatic marker, DLK (20), and the pluripotent progenitor cell marker, CD34 (21), were readily detectable in the HP14.5d cells and minimally detected in the LC $3 \mathrm{~m}$ and Hepa1-6 cells. The tumor marker, AFP, the fetal form of serum albumin (22) and CK19, a non-specific marker for liver stem cells (23), were expressed at higher levels in the HP14.5d cells compared with the LC $3 \mathrm{~m}$ cells. Albumin and CK18, as liver-specific markers, were expressed at lower levels in the HP14.5d cells compared with the LC $3 \mathrm{~m}$ and Hepa1-6 cells. The expression of the mature hepatic markers, apolipoprotein B (ApoB), tyrosine aminotransferase (TAT) and UGT1A (24-26), was undetectable in the HP14.5d cells. Taken together, these results suggest that the LC3m and HP14.5d cells represent mature hepatocytes and HPCs, respectively.

Induction of hepatic differentiation. We then wished to investigate the differentiation potential of the 2 candidate cell lines. The combination of $2 \% \mathrm{HS}+0.1 \mu \mathrm{M}$ Dex $+10 \mathrm{ng} / \mathrm{ml} \mathrm{HGF}+$
$20 \mathrm{ng} / \mathrm{ml} \mathrm{FGF4}$ has been demonstrated to induce the differentiation of progenitor cells into functional hepatocytes in our previous study (27). In this study, we compared the induction of hepatic differentiation between the 2 candidate cell lines. Through ALB-driven luciferase reporter assay (Fig. 4A), we found that the basal level of ALB-Gluc activity in the LC3m cells was higher than that in the HP14.5d cells. As culture time progressed without induction, the ALB-Gluc activity slightly increased in the HP14.5 cells but not in the LC $3 \mathrm{~m}$ cells. ALB-Gluc activity was significantly increased after the induction of differentiation. At 12 days of induction, the ALB-Gluc activity of the HP14.5d cells was comparable to that of LC3m cells. The mRNA expression levels of DLK, CD34, AFP, ALB, TAT and ApoB in the induced HP14.5d cells were similar to those observed in the induced LC3m cells (Fig. 4B). Western blot analysis and immunofluorescence staining revealed that the protein expression of UGT1A and CK18 in the induced HP14.5d cells was increased to levels equivalent to those observed in the same induced LC3m cells (Fig. 4C and D). Thus, our results demonstrate that the HP14.5d cells can easily be induced to differentiate into hepatocytes with an expression profile of hepatic-related markers similar to that of mature hepatocytes.

Function of induced cells. The evaluation of ICG uptake is a common way to estimate liver function (28). As mature hepato- 

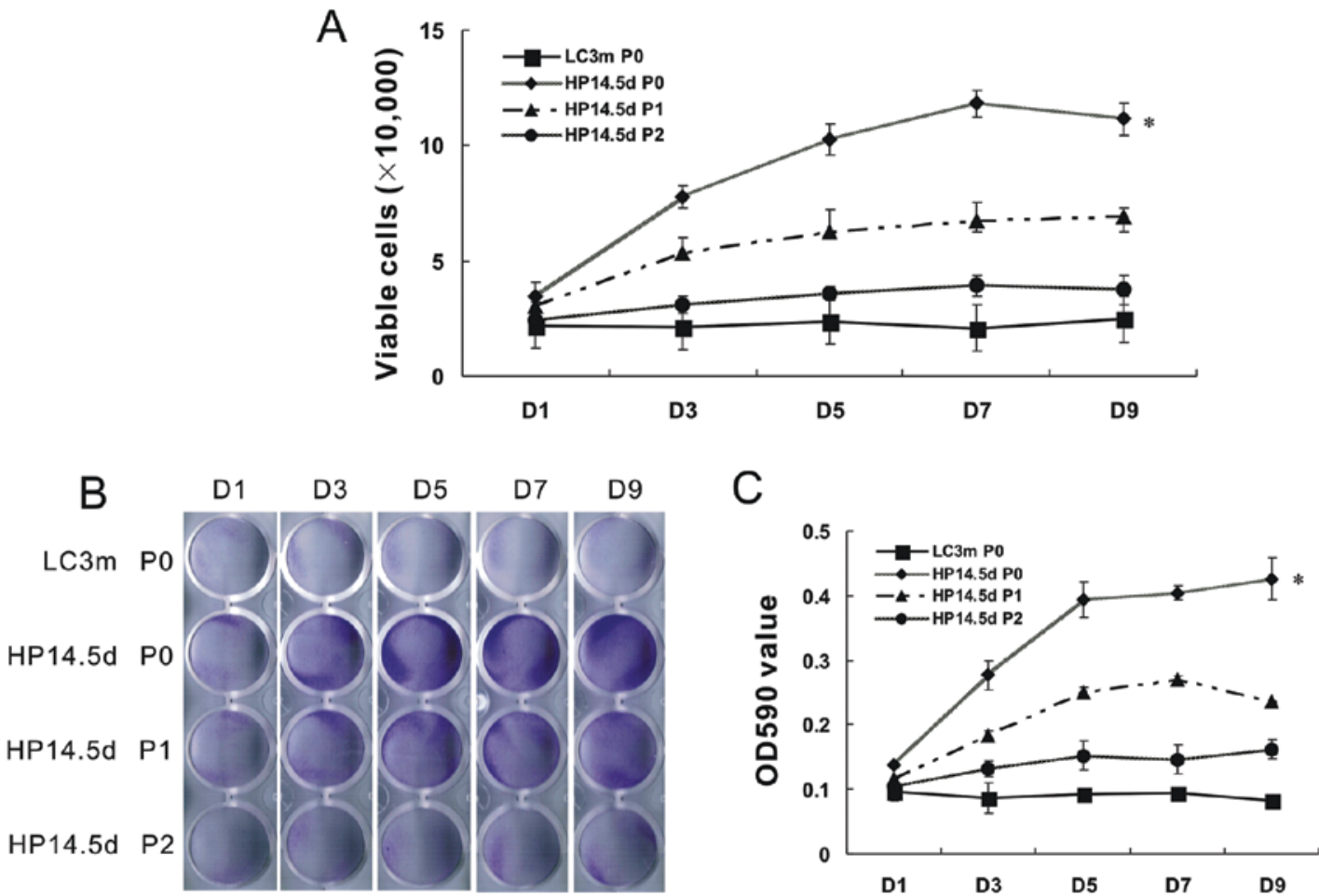

Figure 2. Cell proliferation of primary and passaged cells. (A) Cell growth curve was measured by trypan blue staining to measure viable cells. (B) Crystal violet staining. Cells were stained with crystal violet solution for $10 \mathrm{~min}$. Three independent experiments were performed in duplicate, and representative results are shown. (C) Crystal violet dye of each well as the same set of cells in (B) was dissolved and absorbance was measured at 540 nm (OD540). "P<0.05, HP14.5d cells at P0 vs. LC3m cells; P>0.05, HP14.5d cells at P2 vs. LC3m cells. D, day; P, passage.
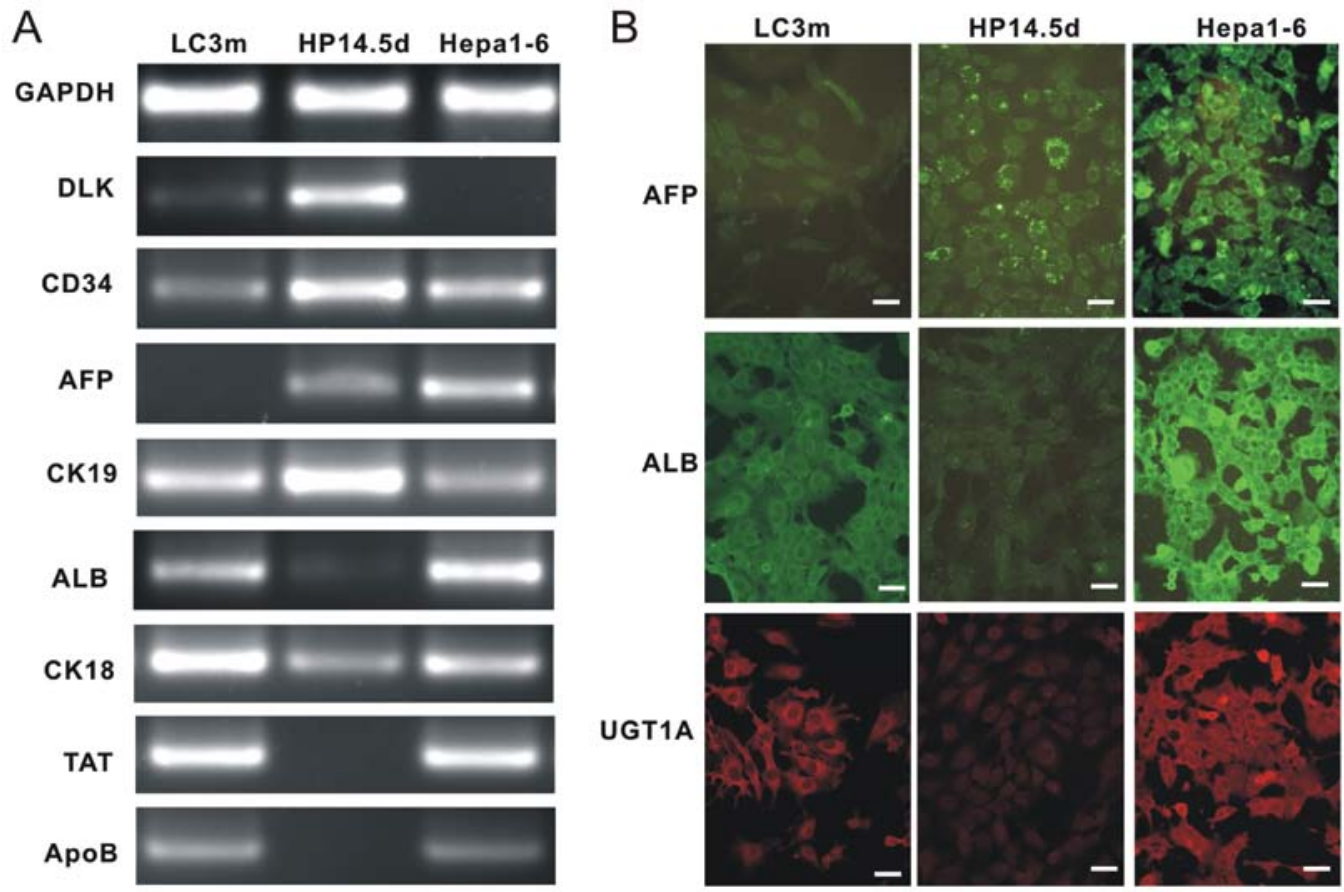

Figure 3. Identification of LC3m cells and HP14.5d cells. Hepa1-6 cells were used as the controls. (A) RT-PCR analysis of the hepatic-related genes, DLK, CD34, AFP, CK19, ALB, CK18, TAT and ApoB. The RT-PCR results were confirmed in at least 3 batches of independent experiments, and representative results are shown. (B) Immunofluorescent staining of AFP, ALB and UGT1A markers. Scale bar, $200 \mu \mathrm{m}$.

cytes, approximately $30 \%$ of the LC3m cells absorbed ICG and exhibited a green-stained nucleus, whereas no ICG-positive cells were observed in the untreated HP14.5d cells. Following
12 days of induction, the ratio of ICG-positive cells in both groups increased to $60 \%$, even though the green color in the HP14.5d cells was lighter than that in the LC3m cells (Fig. 5A). 

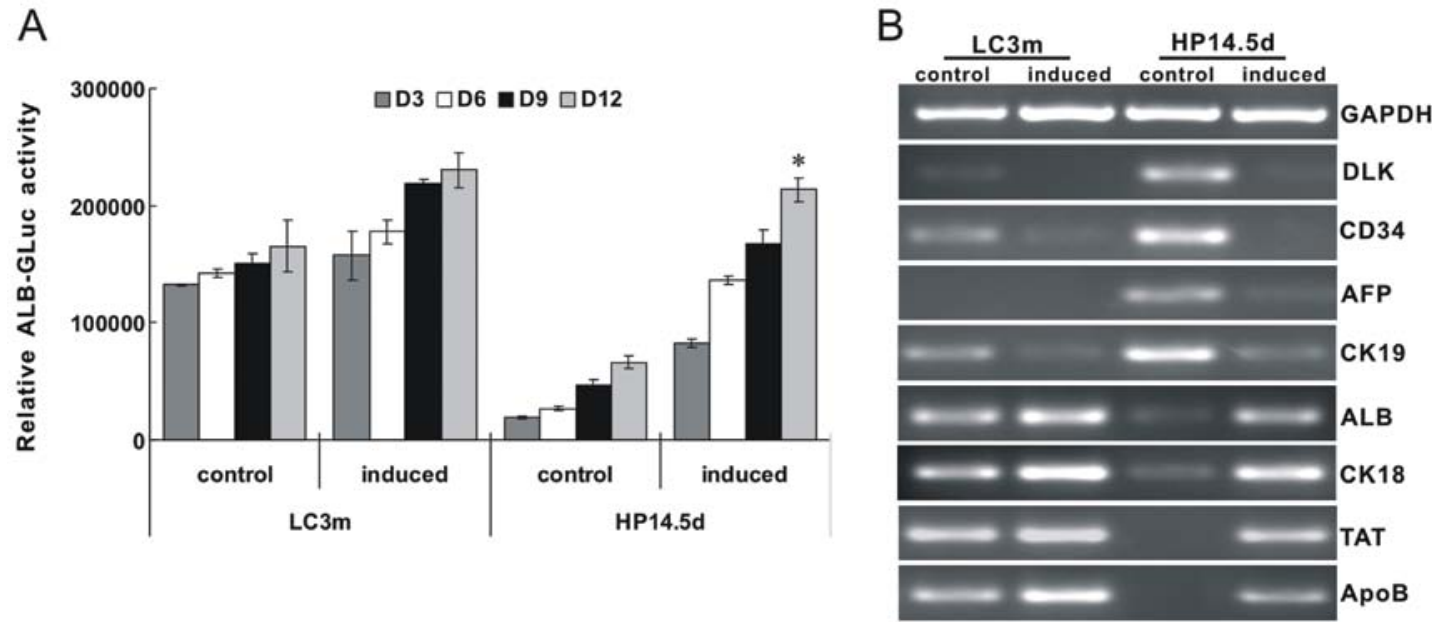

C

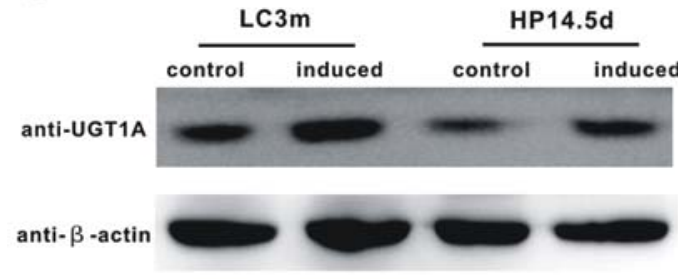

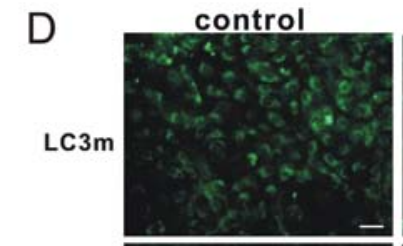

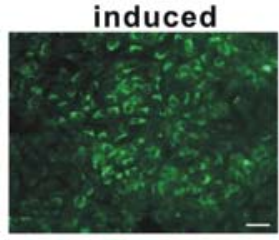

HP14.5d
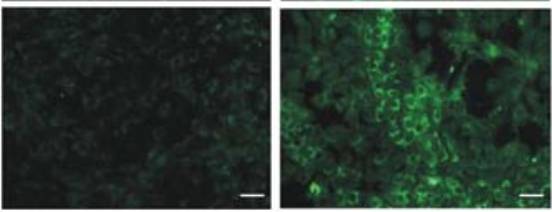

Figure 4. Hepatic differentiation of LC3m cells and HP14.5d cells. Cells were treated with hepatic induction medium for 12 days (induced), untreated cells were set up as the controls. (A) Relative ALB-Gluc activity. Cells were transfected with pSEB-ALB-Gluc plasmid at $48 \mathrm{~h}$ prior to hepatic induction. Medium was collected at the indicated time point and Gaussia luciferase acitivity was measured. ("P $<0.05$ vs. control group; P $>0.05$, induced HP14.5d cells on day 12 vs. induced LC3m cells on day 12). (B) RT-PCR analysis of hepatic-related genes. On day 12 of culture in hepatic induction medium, total RNA of LC3m and HP14.5d cells was extracted; at least 3 batches of independent RT-PCR experiments were performed to detect the expression of DLK, CD34, AFP, CK19, ALB, CK18, TAT and ApoB and representative results are shown. (C) Protein level of UGT1A was detected by western blot analysis on day 12 of treatment in hepatic induction medium. (D) Expression of CK18 was detected by immunofluoresence on day 12 of induction.

A

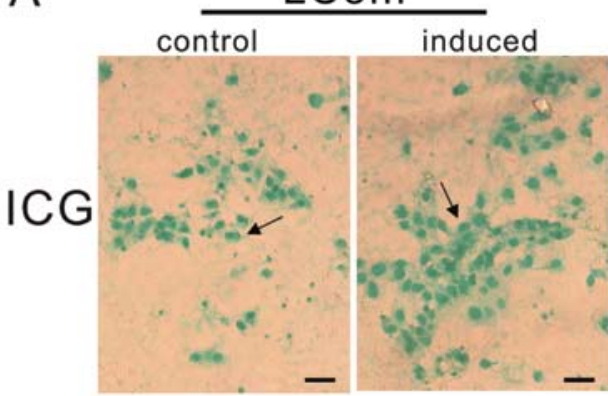

B

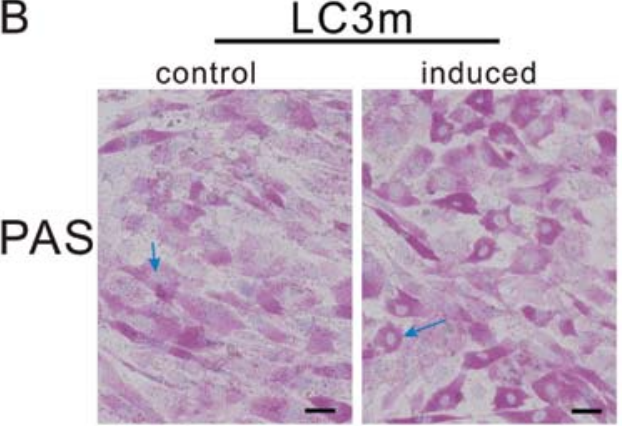

HP14.5d
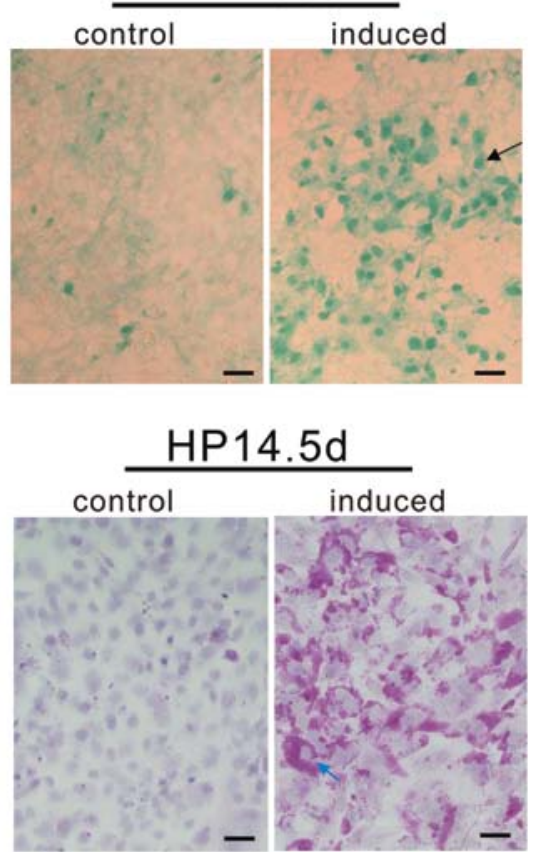

Figure 5. Induced HP14.5d cells displayed functions comparable to those of the LC3m cells. Function analysis was performed on day 12 following treatment in hepatic induction medium (induced cells). (A) Indocyanine green (ICG) uptake assay. Cells with a green-stained nucleus are the positive-stained cells (indicated by black arrows). (B) Periodic acid-Schiff (PAS) staining. Purple color in cell plasma indicates glycogen accumulation (indicated by blue arrows). Scale bar, $200 \mu \mathrm{m}$. 
Mature hepatocytes have the ability of glycogen synthesis and storage. The PAS staining method is used for the detection of glycogen which is displayed by a purple color in the cytoplasm (29). The majority of LC3m cells was positive for PAS staining, and a deeper purple color was observed in the induced LC3m cells. Undifferentiated HP14.5d cells were not stained; however, following treatment in the hepatic induction medium, the number of PAS-stained cells significantly increased and was equivalent to the number of LC3m-stained cells (Fig. 5B). The abovementioned results indicate that the induced HP14.5d cells not only express hepatic markers but also display functions similar to those of mature hepatocytes.

\section{Discussion}

Compared with the strategies of liver transplantation in the treatment of liver diseases, cell-based transplantation therapies have the advantages of low-risk clinical operation, low immunogenicity and good functional recovery, showing tremendous potential for their clinical application $(6-8,30,31)$. The cell source for liver cell transplantation should have the characteristics of effective proliferation capabilities to obtain an adequate population of cells, as well as hepatic functions to replace the damaged liver mass $(13,32)$. To date, a variety of cell sources have been reported in the exploration for liver cell transplantation. Mature hepatocytes have good metabolism functions, but their low availability and expansion efficiency in vitro are major issues of hepatocyte transplantation $(9,33)$. Stem cells have the characteristics of self-renewal, multi-potential differentiation and easy amplification in vitro; however, their limited differentiation capabilities to a hepatic lineage restrict their functional recovery $(34,35)$. HPCs have the ability of bipotential differentiation into mature hepatocytes and biliary epithelial cells, along with self-renewal capacity. In addition, the immunogenicity of HPCs derived from embryos is lower than that of HPCs derived from adult livers, demonstrating their potential for use in clinical practice (36-38). In the present study, we investigated the proliferation, differentiation and function of HPCs compared with mature liver cells, to identify the potential value of HPCs.

As 3-month-old mice are at the adult period of their life-span, the majority of liver cells are at a terminally differentiated stage to perform normal functions. At this stage, primary LC $3 \mathrm{~m}$ cells hardly proliferated and could not be passaged in vitro. Hepatoblasts begin to differentiate into hepatocytes and cholangiocytes on embryonic day 14 (E14) of mouse liver development (39). In this study, we isolated HPCs from the livers of mice on post coitus day 14.5. The majority of the HP14.5d cells belong to stem/progenitor cells, and the freshly isolated HPCs exhibited active proliferation. Following passage, however, their growth capability significantly decreased, and the cells displayed different morphologies. This may due to the various cell types found in liver tissue, such as hepatic parenchymal cells, stellate cells, Kupffer cells and liver fibroblasts (40). It is conceivable that the cell pool may contain HPCs with different proliferative capacities, differentiation potentials or may even have bipotential capabilities. Thus, to obtain a reliable cell source for liver cell transplantation, the characterization and identification of cellular candidates should be further examined. As a following step, we aim to establish individual progenitor clones.
Hepa1-6 is a hepatocarcinoma cell line which expresses high levels of AFP and ALB (41). Using Hepa1-6 cells as the controls, we found that LC $3 \mathrm{~m}$ cells had high expression levels of the mature hepatic marker genes, ALB, CK18, ApoB, TAT and UGT1A (24-26), while the HP14.5d cells exhibited relative high expression levels of pluripotent progenitor cell markers or early hepatic marker genes (DLK, CD34, AFP and CK19) (20-23), suggesting that $\mathrm{LC} 3 \mathrm{~m}$ cells have the genetic characteristics of mature hepatocytes, while the HP14.5d cells retain most, if not all of the HPC phenotype. Even though it has been reported that stem/progenitor cells can replace mature liver cells in cell transplantation, their differentiation and function should be detected prior to their in vivo application. In the present study, we used the combination of $2 \% \mathrm{HS}+0.1 \mu \mathrm{M} \mathrm{Dex}+10 \mathrm{ng} / \mathrm{ml}$ $\mathrm{HGF}+20 \mathrm{ng} / \mathrm{ml} \mathrm{FGF} 4$ to induce the hepatic differentiation of 2 candidate cell lines (27). ALB-driven Gluc activity can indirectly reflect the expression level of ALB, which is used to dynamically measure the hepatic differentiation capalities of liver cells $(18,27)$. The slight increased in ALB-Gluc activity in the untreated HP14.5d cells, indicated the spontaneous differentiation of progenitor cells. Following culture in hepatic induction medium, the expression of hepatic stem/ progenitor and late marker genes in the HP14.5d cells reached levels comparable to those in the induced LC3m cells. Of note, though the LC3m cells were derived from adult mouse livers, ALB-Gluc activity and the expression of late hepatic markers increased following hepatic induction, which suggested that not all cells in the LC $3 \mathrm{~m}$ pool were at a mature status. Nevertheless, we demonstrated the differentiation potential of the HP14.5d cells into mature hepatocytes in vitro.

Synthesis and metabolism are the most important functions of the liver. To be a reliable cell source for cell transplantation, the candidate cell should have normal functions. As a fluorescent dye, ICG is metabolized microsomally in the liver and is only excreted from the circulation by the liver and bile ducts, this characteristic is commonly used as an indicator substance in hepatic function diagnostics. The liver is responsible for glycogenesis (the formation of glycogen from glucose) $(42,43)$. In this study, we performed ICG uptake analysis and PAS staining to detect the function of ICG metabolism and glycogen synthesis/storage, respectively. The untreated HP14.5d cells did not have any hepatic functions, whereas almost $100 \%$ of the LC $3 \mathrm{~m}$ cells were stained positive following PAS staining; only approximately $30 \%$ of the LC3m cells were ICG-positive. ICG green-stained cells gathered together, indicating that ICG metabolism may be involved in the confluence and co-operation among cells. On day 12 following culture in hepatic induction medium, the HP14.5d cells showed a positive ratio of ICG- and PAS-stained cells similar to that of the LC3m cells. However, the green or purple color of the HP14.5d cells was lighter than that of the LC3m cells, and the PAS staining was not distributed in the whole area of the cell plasma. As a result, HPCs performed some hepatic functions following induction, although not at the exact same levels as mature hepatocytes. The treatment time and different induction methods should be further investigated.

In conclusion, we isolated HPCs from embryonic livers and compared their proliferation, differentiation and function with mature liver cells. HPCs displayed active growth capability, good differentiation potential and normal function following treatment in hepatic induction medium, showing an alternative 
to mature liver cells in the cell-based transplantation strategy for liver disease $(14,44)$. However, HPCs still display limited proliferation capabilities which continue to weaken following passage. Therefore, in future studies, immortalized HPCs should be constructed and in vivo models should be used to further evaluate the effectiveness of HPCs and mature liver cells in cell transplantation for the treatment of liver diseases $(45,46)$.

\section{Acknowledgements}

This study was supported by grant from the National Natural Science Foundation of China (no. 81100309 to Y.B.).

\section{References}

1. Clavien PA, Lesurtel M, Bossuyt PM, Gores GJ, Langer B and Perrier A: OLT for HCC Consensus Group: Recommendations for liver transplantation for hepatocellular carcinoma: an international consensus conference report. Lancet Oncol 13: e11-e22, 2012.

2. Samuel D, Colombo M, El-Serag H, Sobesky R and Heaton N: Toward optimizing the indications for orthotopic liver transplantation in hepatocellular carcinoma. Liver Transpl 17 (Suppl 2): S6-S13, 2011.

3. Sze YK, Dhawan A, Taylor RM, Bansal S, Mieli-Vergani G, Rela $\mathrm{M}$ and Heaton N: Pediatric liver transplantation for metabolic liver disease: experience at King's College Hospital. Transplantation 87: 87-93, 2009.

4. Grant D, Fisher RA, Abecassis M, McCaughan G, Wright L and Fan ST: Should the liver transplant criteria for hepatocellular carcinoma be different for deceased donation and living donation? Liver Transpl 17 (Suppl 2): S133-S138, 2011.

5. Katyal S, Oliver JH III, Buck DG and Federle MP: Detection of vascular complications after liver transplantation: early experience in multislice CT angiography with volume rendering. AJR Am J Roentgenol 175: 1735-1739, 2000.

6. Lacaille F: Liver transplantation and liver cell transplantation. Clin Res Hepatol Gastroenterol 36: 304-307, 2012.

7. Lee SW, Wang X, Chowdhury NR and Roy-Chowdhury J: Hepatocyte transplantation: state of the art and strategies for overcoming existing hurdles. Ann Hepatol 3: 48-53, 2004.

8. Sancho-Bru P, Najimi M, Caruso M, Pauwelyn K, Cantz T, Forbes S, Roskams T, Ott M, Gehling U, Sokal E, Verfaillie CM and Muraca M: Stem and progenitor cells for liver repopulation: can we standardise the process from bench to bedside? Gut 58 : 594-603, 2009.

9. Ito H, Kamiya A, Ito K, Yanagida A, Okada K and Nakauchi H: In vitro expansion and functional recovery of mature hepatocytes from mouse adult liver. Liver Int 32: 592-601, 2012.

10. Gaudio E, Carpino G, Cardinale V, Franchitto A, Onori P and Alvaro D: New insights into liver stem cells. Dig Liver Dis 41: 455-462, 2009.

11. Hughes RD, Mitry RR and Dhawan A: Current status of hepatocyte transplantation. Transplantation 93: 342-347, 2012

12. Souza BS, Nogueira RC, de Oliveira SA, de Freitas LA, Lyra LG, Ribeiro dos Santos R, Lyra AC and Soares MB: Current status of stem cell therapy for liver diseases. Cell Transplant 18: 1261-1279, 2009.

13. Oertel M and Shafritz DA: Stem cells, cell transplantation and liver repopulation. Biochim Biophys Acta 1782: 61-74, 2008.

14. Gennero L, Mortimer P, Sperber K, Carloni G and Ponzetto A: Stem cells: an alternative to organ transplantation in chronic degenerative and infectious diseases? New Microbiol 29: 151-167, 2006.

15. Dollé L, Best J, Mei J, Al Battah F, Reynaert H, van Grunsven LA and Geerts A: The quest for liver progenitor cells: a practical point of view. J Hepatol 52: 117-129, 2010.

16. Russo FP and Parola M: Stem and progenitor cells in liver regeneration and repair. Cytotherapy 13: 135-144, 2011.

17. Huang E, Bi Y, Jiang W, Luo X, Yang K, Gao JL, Gao Y, Luo Q, Shi Q, Kim SH, Liu X, Li M, Hu N, Liu H, Cui J, Zhang W, Li R, Chen X, Shen J, Kong Y, Zhang J, Wang J, Luo J, He BC, Wang H, Reid RR, Luu HH, Haydon RC, Yang L and He TC: Conditionally immortalized mouse embryonic fibroblasts retain proliferative activity without compromising multipotent differentiation potential. PLoS One 7: e32428, 2012.
18. Bi Y, Huang J, He Y, Zhu GH, Su Y, He BC, Luo J, Wang Y, Kang Q, Luo Q, Chen L, Zuo GW, Jiang W, Liu B, Shi Q, Tang M, Zhang BQ, Weng Y, Huang A, Zhou L, Feng T, Luu HH, Haydon RC, He TC and Tang N: Wnt antagonist SFRP3 inhibits the differentiation of mouse hepatic progenitor cells. J Cell Biochem 108: 295-303, 2009.

19. Bi Y, Gong M, Zhang X, Zhang X, Jiang W, Zhang Y, Chen J, Liu Y, He TC and Li T: Pre-activation of retinoid signaling facilitates neuronal differentiation of mesenchymal stem cells. Dev Growth Differ 52: 419-431, 2010.

20. Nishina H: hDlk-1: a cell surface marker common to normal hepatic stem/progenitor cells and carcinomas. J Biochem 152: $121-123,2012$.

21. Nyamath P, Alvi A, Habeeb A, Khosla S, Khan AA and Habibullah CM: Characterization of hepatic progenitors from human fetal liver using CD34 as a hepatic progenitor marker. World J Gastroenterol 13: 2319-2323, 2007.

22. Sell S: Alpha-fetoprotein, stem cells and cancer: how study of the production of alpha-fetoprotein during chemical hepatocarcinogenesis led to reaffirmation of the stem cell theory of cancer. Tumour Biol 29: 161-180, 2008.

23. He $\mathrm{Z}$ and Feng M: Activation, isolation, identification and culture of hepatic stem cells from porcine liver tissues. Cell Prolif 44: 558-566, 2011.

24. Pan RL, Chen Y, Xiang LX, Shao JZ, Dong XJ and Zhang GR: Fetal liver-conditioned medium induces hepatic specification from mouse bone marrow mesenchymal stromal cells: a novel strategy for hepatic transdifferentiation. Cytotherapy 10: 668-675, 2008.

25. Tirnitz-Parker JE, Tonkin JN, Knight B, Olynyk JK and Yeoh GC: Isolation, culture and immortalisation of hepatic oval cells from adult mice fed a choline-deficient, ethionine-supplemented diet. Int J Biochem Cell Biol 39: 2226-2239, 2007.

26. Snykers S, Vanhaecke T, Papeleu P, Luttun A, Jiang Y, Vander Heyden Y, Verfaillie C and Rogiers V: Sequential exposure to cytokines reflecting embryogenesis: the key for in vitro differentiation of adult bone marrow stem cells into functional hepatocyte-like cells. Toxicol Sci 94: 330-341, 2006.

27. He Y, Zhang WY, Gong M, Huang JY, Tang N, Feng T, Wei GH, $\mathrm{He}$ TC and Bi Y: Low serum concentration facilitates the differentiation of hepatic progenitor cells. Saudi Med J 32: 128-134, 2011.

28. Yamada T, Yoshikawa M, Kanda S, Kato Y, Nakajima Y, Ishizaka $S$ and Tsunoda $Y$ : In vitro differentiation of embryonic stem cells into hepatocyte-like cells identified by cellular uptake of indocyanine green. Stem Cells 20: 146-154, 2002.

29. Kamo N, Yasuchika K, Fujii H, Hoppo T, Machimoto T, Ishii T, Fujita N, Tsuruo T, Yamashita JK, Kubo H and Ikai I: Two populations of Thy1-positive mesenchymal cells regulate in vitro maturation of hepatic progenitor cellspopulations of Thyl-positive mesenchymal cells regulate in vitro maturation of hepatic progenitor cells. Am J Physiol Gastrointest Liver Physiol 292: G526-G534, 2007.

30. Enns GM and Millan MT: Cell-based therapies for metabolic liver disease. Mol Genet Metab 95: 3-10, 2008.

31. Fitzpatrick E, Mitry RR and Dhawan A: Human hepatocyte transplantation: state of the art. J Intern Med 266: 339-357, 2009.

32. Zhang Z, Liu J, Liu Y, Li Z, Gao WQ and He Z: Generation, characterization and potential therapeutic applications of mature and functional hepatocytes from stem cells. J Cell Physiol 228: 298-305, 2013.

33. Risal P, Cho BH, Sylvester KG, Kim JC, Kim HT and Jeong YJ: The establishment and characterization of immortal hepatocyte cell lines from a mouse liver injury model. In Vitro Cell Dev Biol Anim 47: 526-534, 2011.

34. Sharma AD, Cantz T, Vogel A, et al: Murine embryonic stem cell-derived hepatic progenitor cells engraft in recipient livers with limited capacity of liver tissue formation. Cell Transplant 17: 313-323, 2008.

35. Tomiyama K, Miyazaki M, Nukui M, Takaishi M, Nakao A, Shimizu N and Huh NH: Limited contribution of cells of intact extrahepatic tissue origin to hepatocyte regeneration in transplanted rat liver. Transplantation 83: 624-630, 2007.

36. Krupnick AS, Balsara KR, Kreisel D, Riha M, Gelman AE, Estives MS, Amin KM, Rosengard BR and Flake AW: Fetal liver as a source of autologous progenitor cells for perinatal tissue engineering. Tissue Eng 10: 723-735, 2004.

37. Machaj EK, Grabowska I, Gajkowska A, Jastrzewska M, Oldak T, Moraczewski J and Pojda Z: Differentiation potential of the fetal rat liver-derived cells. Folia Histochem Cytobiol 43: 217-222, 2005. 
38. Khan AA, Shaik MV, Parveen N, Rajendraprasad A, Aleem MA, Habeeb MA, Srinivas G, Raj TA, Tiwari SK, Kumaresan K, Venkateswarlu J, Pande G and Habibullah CM: Human fetal liver-derived stem cell transplantation as supportive modality in the management of end-stage decompensated liver cirrhosis. Cell Transplant 19: 409-418, 2010.

39. Zhao R and Duncan SA: Embryonic development of the liver. Hepatology 41: 956-967, 2005.

40. Kmieć Z: Cooperation of liver cells in health and disease. Adv Anat Embryol Cell Biol 161: III-XIII, 1-151, 2001.

41. Zhang L, Jiang G, Yao F, He Y, Liang G, Zhang Y, Hu B, Wu Y, $\mathrm{Li} \mathrm{Y}$ and Liu H: Growth inhibition and apoptosis induced by osthole, a natural coumarin, in hepatocellular carcinoma. PLoS One 7: e37865, 2012.

42. Lee HJ, Jung J, Cho KJ, Lee CK, Hwang SG and Kim GJ Comparison of in vitro hepatogenic differentiation potential between various placenta-derived stem cells and other adult stem cells as an alternative source of functional hepatocytes. Differentiation 84: 223-231, 2012.
43. Shin KS, Lee HJ, Jung J, Cha DH and Kim GJ: Culture and in vitro hepatogenic differentiation of placenta-derived stem cells, using placental extract as an alternative to serum. Cell Prolif 43: 435-444, 2010.

44. Haridass D, Narain N and Ott M: Hepatocyte transplantation: waiting for stem cells. Curr Opin Organ Transplant 13: 627-632, 2008.

45. Gong M, Bi Y, Jiang W, Zhang Y, Chen L, Hou N, Liu Y, Wei X, Chen $\mathrm{J}$ and $\mathrm{Li} \mathrm{T}$ : Immortalized mesenchymal stem cells: an alternative to primary mesenchymal stem cells in neuronal differentiation and neuroregeneration associated studies. J Biomed Sci 18: 87, 2011.

46. Kakinuma S, Nakauchi $\mathrm{H}$ and Watanabe M: Hepatic stem/ progenitor cells and stem-cell transplantation for the treatment of liver disease. J Gastroenterol 44: 167-172, 2009. 